\title{
BMJ Open Effects of a peer co-facilitated educational programme for parents of children with ADHD: a feasibility randomised controlled trial protocol
}

Ingunn Mundal (D , , ${ }^{1,2,3}$ Rolf W Gråwe, ${ }^{1,4}$ Hege Hafstad, ${ }^{5}$ Carlos De las Cuevas, ${ }^{6,7}$ Mariela Loreto Lara-Cabrera ${ }^{1,8,9}$

To cite: Mundal I, Gråwe RW, Hafstad $\mathrm{H}$, et al. Effects of a peer co-facilitated educational programme for parents of children with ADHD: a feasibility randomised controlled trial protocol. BMJ Open 2020;10:e039852. doi:10.1136/ bmjopen-2020-039852

- Prepublication history for this paper is available online. To view these files, please visit the journal online (http://dx.doi org/10.1136/bmjopen-2020039852).

Received 27 April 2020 Revised 19 August 2020 Accepted 02 November 2020

Check for updates

(C) Author(s) (or their employer(s)) 2020. Re-use permitted under CC BY-NC. No commercial re-use. See rights and permissions. Published by BMJ.

For numbered affiliations see end of article.

Correspondence to Dr Ingunn Mundal; ingunn.p.mundal@ntnu.no

\section{ABSTRACT}

Introduction Significant numbers of children with attention deficit hyperactivity disorder (ADHD) display problems that cause multiple disabilities, deficits and handicaps that interfere with social relationships, development and school achievement. They may have multiple problems, which strain family dynamics and influence the child's treatment. Parent activation, described as parents' knowledge, skills and confidence in dealing with their child's health and healthcare, has been shown to be an important factor in improving health outcomes. Research suggests that parents need edification to learn skills crucial to the treatment and management of their children's healthcare. Promoting positive parenting techniques may reduce negative parenting factors in families. This study aims to assess the acceptability, feasibility and estimated sample size of a randomised controlled trial (RCT) comparing an ADHD peer co-led educational programme added to treatment as usual (TAU).

Methods and analysis Using a randomised waitlist controlled trial, parents of children aged 6-12 years newly diagnosed with ADHD, and referred to a child mental health outpatient clinic in Mid-Norway, will receive TAU concomitant with a peer co-facilitated parental engagement educational programme $(n=25)$. Parents in the control group will receive TAU, and the educational programme treatment within a waitlist period of 3-6 months $(n=25)$. Parent activation, satisfaction, well-being, quality of life and treatment adherence, will be assessed at baseline (T0), 2 weeks (T1) pre-post intervention (T2, T3) and at 3 months follow-up (T4). Shared decision making, parents preferred role in health-related decisions and involvement, parent-reported symptoms of ADHD and child's overall level of functioning will be assessed at T0 and T4. Such data will be used to calculate the required sample size for a full-scale RCT.

Ethics and dissemination Approval was obtained from the Regional Committee for Medicine and Health Research Ethics in Mid-Norway (ref: 2018/1196). The findings of this study are expected to provide valuable knowledge about how to optimise family education and management of ADHD and will be disseminated through presentations at conferences and publication in peer-reviewed journals. Trial registration number NCT04010851.
Strengths and limitation of this study

The intervention is delivered by collaborating health professionals and user representatives.

- This feasibility study will endorse methods, questionnaires and procedures for a subsequent fullscale randomised controlled trial.

- Other attention deficit hyperactivity disorder interventions, including psychoeducation, may contaminate and weaken the validity of the intervention.

- Participant enrolment and drop-out may increase selection and attrition bias, respectively.

\section{INTRODUCTION}

Patient-centred care, shared decisionmaking, patient participation and models of care which incorporate user involvement and patients' perspectives on their treatment and care, are collaborative models entailing that mental health services should emphasise users' needs, users' participation and involvement. ${ }^{1}$ Patient-centred interventions entail comprehensive efforts to empower users in mental health services by increasing the user's capacity to engage with healthcare providers. ${ }^{2}$ Based on this patientcentred framework, an educational intervention aims at improving interactions between users and health professionals. In terms of outcomes, patient-centred approaches focus on improved health outcomes as well-being and quality of life, perceived quality of care, shared decision-making, increased satisfaction with healthcare and improved personal outcomes, such as parental engagement, knowledge acquisition, self-management and confidence. $^{2}$

Parents have a key role in managing their child's mental health problems and treatment. However, many parents experience difficulties and a lack of support in finding information about their child's condition. ${ }^{3}$ 
Parental education, service-user involvement and parental activation may therefore be important elements to provide adequate treatment information and support to family caregivers. Facilitation of parent involvement in treatment is an ethical requirement in order to provide adequate treatment and support to family caregivers. ${ }^{4}$ Service-user involvement refers to an active partnership between service-users and mental health professionals regarding planning, implementation and evaluation of health services. ${ }^{5}$ Effective treatment of children requires optimal communication, sharing of information and shared decision-making between health professionals and the family. ${ }^{6}$

Attention deficit hyperactivity disorder (ADHD) is the most common childhood neuropsychiatric disorder, ${ }^{78}$ with an average prevalence of $5 \%{ }^{9}$ and endures into adulthood in $30 \%-60 \%$ of inflicted cases. ${ }^{10}{ }^{11}$ Core symptoms of inattention, hyperactivity and impulsivity are frequently associated with comorbid oppositional defiant or conduct disorders ${ }^{12}$ and mood and anxiety disorders. ${ }^{13}$ There is also some overlap between ADHD and substance use disorders, obsessive-compulsive disorder, tics, sleep disorders, specific learning disorders, autistic spectrum disorder and eating disorders. ${ }^{13}$ Many comorbid problems affect their families. ${ }^{14}$ Moreover, parents of these children with psychopathology are at increased risk of having psychiatric symptoms. ${ }^{15}$

Nearly $20 \%$ of children with ADHD display behaviour problems that interfere with their relationship development and academic achievement, ${ }^{16}$ and an early onset of behavioural problems is frequently a sign of persistent problems. ${ }^{17} 18$ In two meta-analytic reviews, parent participation in parent training programmes for ADHD was shown to decrease child problem behaviours and ADHD symptoms, and promote positive parent-child relationships. ${ }^{19}{ }^{20}$ Evidence also suggest that psychoeducational programmes in children and adolescents with $\mathrm{ADHD}^{21}$ have positive outcomes as measured by a significant reduction in core symptoms and inattention levels, ${ }^{22}$ parent and child satisfaction, ${ }^{23}$ children's knowledge of ADHD,${ }^{24}$ children's attitudes towards medication ${ }^{2526}$ and adherence to medical recommendations. ${ }^{27}{ }^{28}$ Yet, the available evidence from this review is limited due to major challenges including differences in the definition of psychoeducation, levels of complexity, degree of diversity of the interventions and simply indicates the positive role of parental education interventions in children and adolescents with ADHD. ${ }^{21}$ A randomised controlled trial (RCT) found that a psychoeducative programme for parents reduced ADHD symptoms both in the short term and at 1 year follow-up. ${ }^{22}$

Overall, most studies show promising effects of the educational intervention delivered by health professionals. Such interventions have shown effects on parent coping, ${ }^{29}{ }^{30}$ increased confidence in the abilities of the parents, increased parenting satisfaction and activation, ${ }^{31-38}$ and are also associated with improvements in child-level and parent-level outcomes. ${ }^{39}$ In addition to traditional health models of educational interventions, recent studies have suggested that peers can lead or co-facilitate group education interventions. A recent pilot study showed the potential effectiveness of peer-to-peer delivered services, and demonstrated that participation in paraprofessional-delivered behavioural parent training to parents of children with ADHD was associated with favourable improvements in child-level and parent-level outcomes. ${ }^{39}$ Another study of a psychoeducational model delivered by community volunteers, found significantly greater improvement in parenting behaviour, parents' perception of competence, child functional impairment, parental stress and depressive symptoms, compared with a waitlist condition. ${ }^{40}$

Although the above-mentioned studies have reported promising effects of parental educational programmes, there is no evidence to support the efficacy of peer co-facilitated educational approaches for parents of children with $\mathrm{ADHD}$, that is, interventions developed and provided through collaboration between user representatives and health personnel. ${ }^{21}$

Peer educators and health professionals may emphasise different values when developing and implementing educational programmes. ${ }^{41}$ Parents who are experts have a unique knowledge about the mental healthcare systems work, and by their experience, they may have important things to teach other parents. The active involvement of parents in educational interventions, incorporating their knowledge to help other parents may therefore be beneficial. This is pertinent as user involvement in planning and delivering educational interventions become embedded in research. ${ }^{42}$

In this study, we will assess whether a brief peer co-led parenting programme, developed and provided in collaboration between user representatives and health personnel, would be effective in reducing disruptive behaviour and ADHD symptoms in school-aged children. ${ }^{43-45}$

\section{Aims and objectives}

This study aims to assess the acceptability, feasibility and estimated sample size in an RCT, comparing an ADHD peer co-led educational programme conducted alongside treatment as usual (TAU). The educational sessions are planned and co-led by peer educators and involve a sample of parents of children and adolescents living in Mid-Norway who have been diagnosed with ADHD. The treatment is generally available for parents in hospitals in the Mid-Norway Hospital Trust.

The primary objective of this trial is to evaluate the acceptability and feasibility of the intervention primarily with respect to practical issues: (1) whether parents of children recently diagnosed with ADHD are willing to be randomly assigned to the intervention, (2) whether sufficient numbers of families can be recruited and retained such that a full-scale RCT is likely to be feasible, (3) whether research procedures and efficacy measures are feasible and acceptable to participating families and the 
outpatient clinics, (4) and whether families participating in the intervention are satisfied with the programme. The sample used is not powered to detect differences in major clinical endpoints.

\section{METHODS AND ANALYSES}

This pilot RCT will evaluate the feasibility and acceptability of delivering and evaluating a peer co-facilitated educational group to inform a larger-scale randomised study that will allow for a more rigorous assessment of the impact of the intervention. ${ }^{46-48}$ We will explicitly investigate whether parents are willing to participate in a feasibility research evaluation, exploring acceptability (assessing attendance and client satisfaction) and reasons for ineligibility, as well as the retention of the study (including baseline, intervention and a 3-month follow-up). Additionally, we will investigate the most appropriate primary outcome (informed by qualitative feedback, feasibility data, SD and effect size estimates) to ensure if the method, questionnaires and procedures are adequate for a subsequent full-scale RCT. Measurements of the children will be conducted at baseline (T0) and 3 months follow-up (T4), and of the parents at baseline, 2 weeks (T1), pre-post intervention (T2 and T3) and at 3-month follow-up (T4). Included measures address key components of the intervention (activation, quality of life, psychological well-being, parent satisfaction and shared decision-making).

Feasibility assessments will include:

- Recruitment issues.

- Acceptability of randomisation and procedures indicated by loss to follow-up (pretest to post-test and 3-month follow-up) and reasons for withdrawal.

- Satisfaction with the programme.

Feasibility of quantitative measures will be deemed acceptable if no questionnaires are missing for more than $25 \%$ of the participants, and if Cronbach's $\alpha$ is higher than 0.70 and assessed by follow-up response rates (preintervention to post-intervention and 3-month follow-ups). Methods in this study will adhere to the Standard Protocol Items Recommendations for Interventional
Trials guidelines for the reporting of study protocols. ${ }^{49}$ The study will be conducted between September 2020 and June 2021.

\section{Study design and setting}

This is a feasibility RCT with a parallel assignment as the intervention will be delivered in an educational group format within the child outpatient mental health services (CMHS) in St Olav's Hospital in Mid- Norway. As this feasibility study is not designed to detect a treatment effect, a maximum of 50 parents will be recruited. The trial will consist of two arms, including 25 parents in each arm.

\section{The intervention}

Both the intervention group (IG) and the control group (CG) will receive TAU in addition to psychoeducation or waitlist. Basically, the intervention aims to improve parent activation, and self-management by providing information and knowledge to the parents. The 1 day psychoeducational programme, which is organised and led by user representatives and health professionals, contains five session involving (a) an introduction targeting the rationale for the programme, (b) user representative experiences, (c) a psychiatrist providing information about ADHD (diagnosis, symptoms, perception and treatment), (d) experience from a young user representative with ADHD and (e) a teachers seminar. The user representatives are recruited, approved and trained by the user organisation ADHD Norway and Varres. The group sessions of self-help for parents are arranged by ADHD Norway (parents are invited to register during the course).

A brief summary of the content of the sessions and time schedule is presented in table 1 .

\section{The intervention group (IG)}

Parents $(n=25)$, randomised to the IG will receive the educational programme within 3 months after the randomisation, combined with standard psychosocial and pharmacological care. The intervention will be administered during a full day's training, focusing on ADHD diagnosis and best practice treatment, coping with parenting challenges and experiences with education-related advice.

\begin{tabular}{|c|c|c|c|}
\hline Session & Time schedule & Topics & Responsible lecturer \\
\hline 0 & 08:00-08:30 & Registration & $\begin{array}{l}\text { Health professionals and } \\
\text { user representatives }\end{array}$ \\
\hline 1 & 08:30-08:45 & Welcome and practical information & $\begin{array}{l}\text { Health professionals and } \\
\text { user representatives }\end{array}$ \\
\hline 4 & $12: 45-13: 15$ & Living with ADHD & User representative \\
\hline 5 & $13: 30-15: 30$ & A whole life: actions in kindergarten, school, home & Teacher \\
\hline
\end{tabular}

ADHD, attention deficit hyperactivity disorder. 
After participating in the 1 day intervention, parents will be able to continue in two self-help group sessions (2-hour evening session) lead by user representatives. Participation does not require fees and aim to offer practical tools, support and information to increase parents' skills, knowledge and confidence. Possible effects of the intervention will be assessed by examining differences between the intervention and control group throughout the follow-up period.

\section{The control group (CG)}

Parents $(n=25)$, randomised to the CG (TAU and the same educational programme, but according to a waitlist period and within 3-6 months), will receive standard psychosocial and pharmacological care between baseline and the 6-month follow-up.

\section{Eligibility and recruitment}

A maximum of 50 consenting parents of children, newly diagnosed with ADHD, will be invited to participate in the study by a health professional who administers a waitlist of parents interested in attending a parental education group. The invited parents will be asked to participate in the study through an open invitation brochure providing information about the aim of the project and a response option to the project manager. Those who are interested in participating will be invited to an individual inclusion appointment. All participants must provide written informed consent followed by randomisation and baseline questionnaires. Inclusion criteria are (1) parent/ caregiver of a child with a recent diagnosis of ADHD; (2) child age 6-12 years at baseline and (3) parent/caregiver can speak or have a good understanding of a Scandinavian language. An externally administered, internetbased Case Record Form (WebCRF3) will collect research data and randomise the participants to the two trial arms. Allocation concealment will be ensured, and the randomisation code will not be released until the patient has been recruited into the trial after the completion of baseline measurements. ${ }^{50}$ The parents will not receive any restrictions with regard to participation in other concomitant non-educational care and interventions during the trial. The participants may contact the project manager if they have questions concerning the attendance, the trial procedures, the security of the personal data or if they need assistance. The recruitment procedure and assignments are presented in figure 1.

The study will be reported in accordance with the Template for Interventions Description and Replication and the Consolidated Standards of Reporting Trials.

\section{Sample size and statistical power calculation}

The sample size in this study is related to the number of patients required for enrolment, opinions about the questionnaires and satisfaction with the intervention.

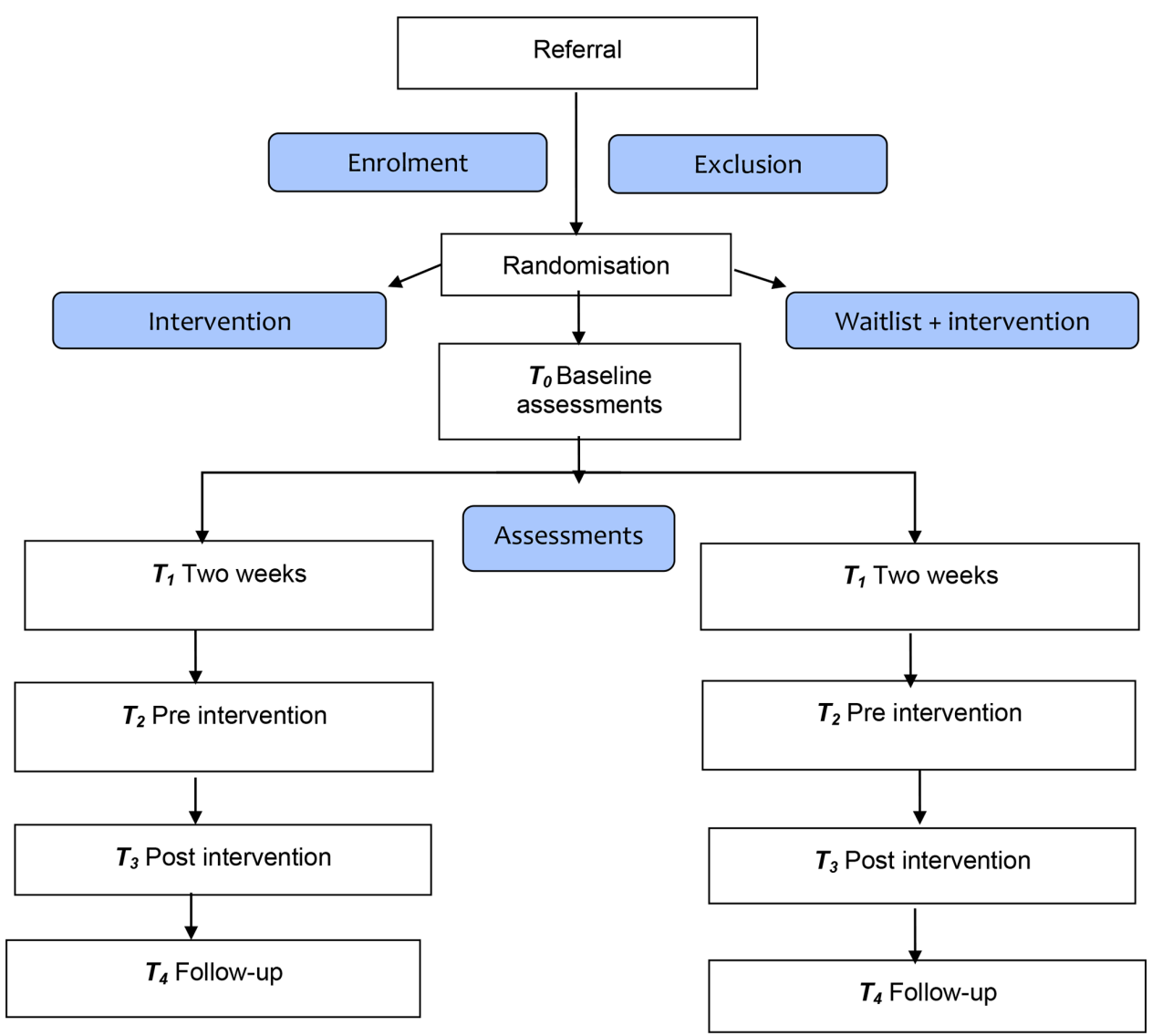

Figure 1 Flow diagram illustrating recruitment and assessments of participants through the study. Adapted from SPIRIT 2013 Statement: Defining Standard Protocol Items for Clinical Trials Consolidated. 
Consequently, the anticipated sample will be 50 parents, that is, 25 parents in each arm.

\section{Measures}

Feasibility assessments and criteria for feasibility success:

- Feasibility of recruitment will be considered acceptable if $50 \%$ of all eligible parents can be recruited.

- Acceptability of randomisation and procedures will be determined by measuring loss to follow-up (from pretest to post-test). An acceptable attrition rate is considered to be $30 \%$. Reasons for withdrawal will be collected.

- Feasibility of quantitative measures will be deemed acceptable if no questionnaires are missing for more than $25 \%$ of the participants, and if Cronbach's $\alpha$ is higher than 0.70 .

- Follow-up response rates (postintervention and 3-month follow-up) will be assessed by monitoring possible attrition.

- Participation and attendance (also questionnaire fulfilments) will be ensured by SMS (text message) reminders.

Assessments of parent satisfaction, quality of life, wellbeing and parent activation will serve as indicators of the feasibility outcomes.

- The schedule for enrolment and measurements are listed in figure $2 .{ }^{51}$

\section{Measurements for parents}

Parent Patient Activation Measure (P-PAM)

The P-PAM is adapted from the PAM, which ascertains health engagement and thereby captures parents' selfreported knowledge, skills, behaviours and confidence in the management of their children's health. ${ }^{52}$ The 13-item measure has four possible response options, ranging from (1) strongly disagree to (4) strongly agree, and an additional 'not applicable' option. To calculate the total P-PAM score, the raw score is divided by the number of items answered (excepting non-applicable items) and multiplied by 13. This score is transformed onto a scale with a theoretical range of between 0and 100, based on calibration tables, with a higher P-PAM score indicating greater parental activation. With PAM, an improvement of 4 points is considered a minimal clinically important difference ${ }^{5354}$ We have no comparable studies by means of which to estimate power; however, in a recent study with PAM-Mental Health as an outcome, the Cronbach's $\alpha$ coefficient was reported to be $0.79{ }^{38}$

\section{Client Satisfaction Questionnaire (CSQ-3)}

Parent satisfaction will be measured by means of the CSQ- $3^{55}$ as it applies to the group-based educational programme. The scale comprises three items measured on a scale from 1 (not satisfied) to 4 (very satisfied). A total score of between 4 and 12 indicates the level of satisfaction with the services provided. The CSQ-3 is the short version of CSQ- $-8^{56}$ and represents the three items most salient to the measurement of service satisfaction.
WH0-5 Well-Being Index (WH0-5)

Parental well-being will be measured by the WHO- $5 .{ }^{56}$ The questionnaire has five items rated on a 6-point scale from 0 (all the time) to 5 (at no time), transformed onto a scale from 0 to 100 (high scores indicate better wellbeing). The WHO-5 captures emotional well-being and contains five positively worded items: 'I have felt cheerful and in good spirits', 'I have felt calm and relaxed', 'I have felt active and vigorous', 'I woke up feeling fresh and rested' and 'My daily life has been filled with things that interest me'. Cronbach's $\alpha$ coefficient is reported to be $0.75 .^{56}$

\section{Multicultural Quality of Life Index (MQLI)}

Parental Quality of life will be measured with the 10-item MQLI,${ }^{57}$ which covers key aspects of the concept, from physical well-being to spiritual fulfilment and with a Cronbach's alpha of 0.92 documenting its internal consistency. ${ }^{57}$

\section{Beliefs About Medicines Questionnaire (BMQ) and Medication Adherence Rating Scale}

To investigate beliefs about medicines, we will use the $\mathrm{BMQ}^{5859}$ and Medication Adherence Rating Scale, ${ }^{60}$ which has satisfactory psychometric properties for patients with mental and medical disorders.

\section{Health Beliefs Questionnaire on Psychiatric Treatment—Parents} Version (PHBQ-P)

The parent version of the PHBQ is an adjusted version of the Patient's PHBQ ${ }^{61}$ PHBQ-P integrates the concepts of attitudes towards psychiatric medication, locus of health control and psychological reactance and predicts patient adherence to prescribed medications.

\section{Shared Decision-Making Questionnaire (SDMQ), Control} Preferences Scale and CollaboRATE

The nine-item Shared Decision-Making Questionnaire ${ }^{62}$ will be assessed in order to study the parent-therapeutic relationship regarding parents involvement, control and information over clinical health-related decisions, combined with a modified version of the Control Preferences Scale ${ }^{63}$ and the three-items CollaboRATE ${ }^{64}$ in order to provide information on the parents' preferred role in involvement.

\section{SNAP-IV 18-Item Rating ADHD Scale Parent Version}

The SNAP-IV 18-Item Rating ADHD Scale is an abbreviated version of the Swanson, Nolan and Pelham (SNAP) Questionnaire $^{65}$ and includes items for the two subsets of symptoms-inattention (items 1-9) and hyperactivity/ impulsivity (items 10-18) - from the DSM-IV (Diagnostic and Statistical Manual of Mental Disorders, 4th Edition) criteria for ADHD. The scores in each of the two subsets are added together. The SNAP-IV is used to measure ADHD symptoms and behavioural problems in schoolaged children. Symptom severity is rated on a 4-point scale. There is no evidence of psychometric properties in the Norwegian version of the SNAP-IV. ${ }^{66}$ 


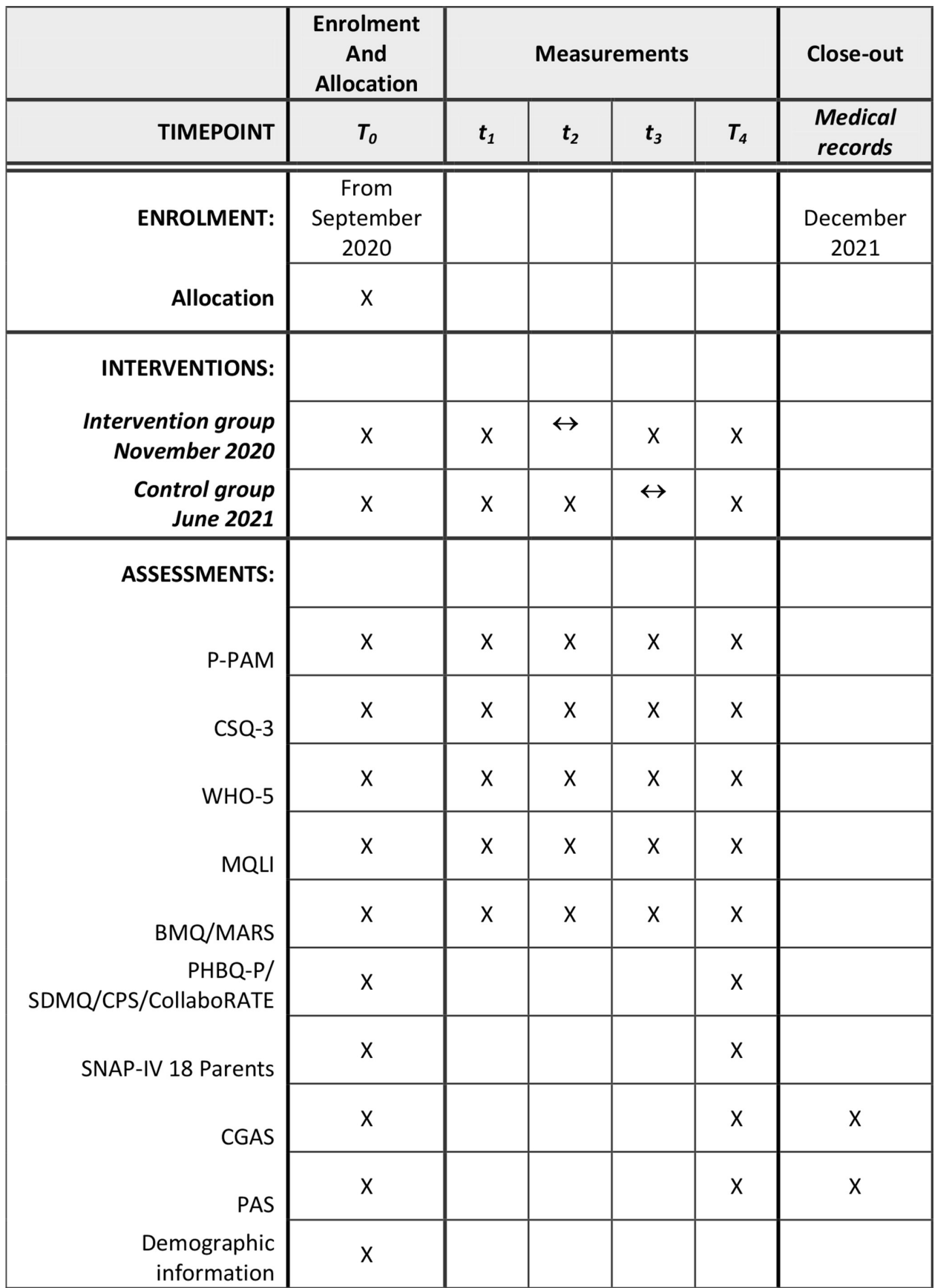

Figure 2 Schedule for enrolment and measurements. Schedule for enrolment, interventions and measurements: Baseline (T0), 2 weeks (T1) pre-post intervention (T2, T3) and at 3-months follow-up (T4). Adapted from SPIRIT 2013 Statement: Defining Standard Protocol Items for Clinical Trials Consolidated. BMQ, beliefs about medicines questionnaire; CGAS, children's global assessment scale; CPS, control preferences scale; CSQ-3, client satisfaction questionnaire; MARS, medication adherence rating scale; MQLI, multicultural quality of life index; PAS, patient administrative system; PHBQ-P, health beliefs questionnaire on psychiatric treatment-parents version; P-PAM, parent patient activation measure; SDMQ, shared decision-making questionnaire; SNAP-IV, swanson, nolan and pelham questionnaire; WHO-5, WHO-5 well-being index.

\section{Measures for children}

Children's Global Assessment Scale (CGAS)

The CGAS is a numeric scale ranging from 1 to 100 that is used to assess the overall level of functioning of children in child and adolescent mental health services, with high scores indicating better functioning. ${ }^{6768}$ The CGAS has favourable psychometric properties, is characterised by its simplicity, and represents an important aspect of assessment in clinical practice and research. The CGAS has predictive value and measures change over time, including treatment effects. It serves as a complement to syndrome-specific scales. ${ }^{69}$

\section{Patient Administrative System}

The Patient Administrative System will be used to collect data about the treatment received, treatment completion, 
percentage of non-attendance in scheduled sessions, participation in collaborative meetings and dropout rates at one and 2 years after the baseline.

\section{Data analyses}

In order to calculate preliminary effects in this feasibility study, $\chi^{2}$ statistics and $t$ tests will be used for descriptive analyses of categorical and continuous variables, respectively, in addition to analysis of variance tests for group comparisons of non-categorical data. Predictors for the preliminary efficacy of the peer co-led educational group will be examined using linear regression on the total sample (pre-post intervention). All included cases will be analysed by an intention-to-treat protocol. No interim analysis is planned. Intervention adherence will be calculated by assessing the response rate of participants receiving the intervention, response rate of returned questionnaires and the number of sessions completed by intervention group participants. Information of absolute and percentage frequencies of recruitment rate and retention rate will be calculated from the data and will be used to inform future definitive RCT design. All statistical analyses will be conducted using SPSS V. 27 and STATA V.16.1.

Data on power estimates will be calculated from the level of improvement of parent activation. An improvement of 4 points or more on the PAM is considered a minimum clinically important difference as this is the level of increase associated with performing a range of self-management behaviours. ${ }^{33}$ Thus, the calculation of the sample size and the minimum number of participants required for each cohort will be based on the SD and the level of improvement in the pilot study. ${ }^{48}$

\section{Ethics and dissemination}

The involvement of user representatives is an overarching principle in our study and includes three components: giving advice and planning the interventions, co-leading the educational group interventions and providing feedback as part of the research team. Thus, the interventions have been codeveloped, conducted and will be implemented in cooperation with user representatives in Mid-Norway.

The Regional Committee for Medical and Health Research Ethics approved the protocol trials for this project (2018/1196-11). This project will be conducted in accordance with the Helsinki Declaration. Participation is voluntary and written informed consent will be signed prior to participation. All parents will follow the normal routines of assessment at the clinic. As the intervention presents no risks or expected harmful effects, there will be no interim analysis. However, if parents want to withdraw from participating in the intervention, they will continue in ordinary treatment without any negative consequences.

The study data will be stored in securely locked files and password-protected databases. Anonymised data will be available for the project leader and the statistician. If the findings from the trials, including user involvement in the research process, document positive effects, the authors' objective is to implement the findings in CMHS in Central Norway and conduct larger-scale studies in the field of ADHD, including studies using health registry data.

To our knowledge, this is the first RCT assessing the efficacy of a peer co-facilitated educational programme for parents of children with ADHD involving educators who are user representatives and parents of children with ADHD. As such, the findings of this study are expected to provide valuable knowledge about how to optimise family education and management of ADHD and will be submitted for publication in a peer-reviewed journal. Advancements achieved with this study, if effective and feasible, will likely be widely disseminated in BMJ dependent on ongoing funding.

\section{Patient and public involvement}

This project has been planned and organised in close collaboration with user representatives from Vårres Regional User Involvement Centre and the ADHD Association, Mid-Norway. User representatives and St Olav University Hospital Patient Education Resource Centre were involved in the design and elaboration and will be involved in the conduct, reporting or dissemination plans of this research.

\section{Author affiliations}

${ }^{1}$ Department of Mental Health, Faculty of medicine and health sciences, Norwegian University of Science and Technology (NTNU), Trondheim, Norway

${ }^{2}$ Faculty of Health and Social Sciences, Molde University College, Molde, Norway ${ }^{3}$ Division of Psychiatry, Kristiansund Community Mental Health Centre, Møre og Romsdal Hospital Trust, Kristiansund, Norway

${ }^{4}$ Division of Psychiatry, Nidaros Community Mental Health Centre, St Olav's University Hospital, Trondheim, Norway

${ }^{5}$ Division of Mid-Norway, Vårres Regional User Involvement Centre, Trondheim, Norway

${ }^{6}$ Department of Internal Medicine, Dermatology and Psychiatry, Universidad de La Laguna, San Cristóbal de La Laguna, Tenerife, Spain

${ }^{7}$ Instituto Universitario de Neurociencia (IUNE), Universidad de La Laguna, San Cristóbal deLa Laguna, Tenerife, Spain

${ }^{8}$ Division of Psychiatry, Tiller Community Mental Health Centre, St Olav's University Hospital, Trondheim, Norway

${ }^{9}$ Department of Research and Development, Division of Mental Health, St Olav's University Hospital, Trondheim, Norway

Acknowledgements The authors thank Anne Karin Kristiansen and Inger Marie Opøien at the Patient Education Resource Centre and the Child and Adolescent Psychiatric Clinic at St Olav's University Hospital, for their work with the development and facilitation of the project. The authors also thank the user representatives Mona Breding Lersveen and Arthur Mandahl from the Vårres organisation, for involvement and input in the planning phase.

Collaborators The study is an active collaboration between the user representatives from VÅRRES, the ADHD Association, Mid-Norway Patient Education Resource Centre, and Child and Adolescent Psychiatric Clinic, St Olav University Hospital, and the Regional Competence Centre for Youth—Mental Health and Child Welfare, Norwegian University of Science and Technology, NTNU, Trondheim, Norway.

Contributors IM, HH and MLL-C conceived the idea for the planned study. RWG, HH and CDIC contributed to drafting the manuscript and revised it critically for important intellectual content. All authors read and provided a substantial contribution to the final version of the study protocol and approved the final version of the manuscript. 
Funding The authors have not declared a specific grant for this research from any funding agency in the public, commercial or not-for-profit sectors.

Competing interests None declared.

Patient and public involvement User representatives and St. Olav University Hospital Patient Education Resource Centre were involved in the design and elaboration, and will be involved in the conduct, reporting or dissemination plans of this research.

\section{Patient consent for publication Not required.}

Provenance and peer review Not commissioned; externally peer reviewed.

Open access This is an open access article distributed in accordance with the Creative Commons Attribution Non Commercial (CC BY-NC 4.0) license, which permits others to distribute, remix, adapt, build upon this work non-commercially, and license their derivative works on different terms, provided the original work is properly cited, appropriate credit is given, any changes made indicated, and the use is non-commercial. See: http://creativecommons.org/licenses/by-nc/4.0/.

ORCID iD

Ingunn Mundal http://orcid.org/0000-0001-7716-7122

\section{REFERENCES}

1 Storm M, Edwards A. Models of user involvement in the mental health context: intentions and implementation challenges. Psychiatr Q 2013;84:313-27.

2 McMillan SS, Kendall E, Sav A, et al. Patient-Centered approaches to health care: a systematic review of randomized controlled trials. Med Care Res Rev 2013;70:567-96.

3 Doody O, Butler MP, Lyons R, et al. Families' experiences of involvement in care planning in mental health services: an integrative literature review. J Psychiatr Ment Health Nurs 2017;24:412-30.

4 Lovdata. Lov Om pasient- OG brukerrettigheter (pasient OG brukerrettighetsloven, 1999Helse- og omsorgsdepartementet. Available: https://lovdata.no/dokument/NL/lov/1999-07-02-63 [Accessed LOV-2020-06-19-79 fra 01.07.2020].

5 Millar SL, Chambers M, Giles M. Service user involvement in mental health care: an evolutionary concept analysis. Health Expect 2016;19:209-21.

6 Melo EMdeOPde, Ferreira PL, Lima RAGde, Melo EM, Lima RA, et al The involvement of parents in the healthcare provided to hospitalzed children. Rev Lat Am Enfermagem 2014;22:432-9.

7 Ferrin M, Perez-Ayala V, El-Abd S, et al. A randomized controlled trial evaluating the efficacy of a Psychoeducation program for families of children and adolescents with ADHD in the United Kingdom: results after a 6-month follow-up. J Atten Disord 2020;24:768-79.

8 Wilens TE, Spencer TJ. Understanding attention-deficit/ hyperactivity disorder from childhood to adulthood. Postgrad Med 2010;122:97-109.

9 Sayal K, Prasad V, Daley D, et al. Adhd in children and young people: prevalence, care pathways, and service provision. Lancet Psychiatry 2018;5:175-86.

10 Mannuzza Set al. Hyperactive boys almost grown up. Arch Gen Psychiatry 1991;48:77-83.

11 Weiss M, Murray C. Assessment and management of attentiondeficit hyperactivity disorder in adults. CMAJ 2003;168:715-22.

12 Biederman J, Monuteaux MC, Mick E, et al. Young adult outcome of attention deficit hyperactivity disorder: a controlled 10-year follow-up study. Psychol Med 2006;36:167-79.

13 Masi L, Gignac M. Adhd and comorbid disorders in childhood psychiatric problems, medical problems, learning disorders and developmental coordination disorder. Clin Psychiatry 2015;1.

14 Ferrin M, Taylor E. Child and caregiver issues in the treatment of attention deficit-hyperactivity disorder: education, adherence and treatment choice. Future Neurol 2011;6:399-413.

15 Wesseldijk LW, Dieleman GC, van Steensel FJA, et al. Risk factors for parental psychopathology: a study in families with children or adolescents with psychopathology. Eur Child Adolesc Psychiatry 2018;27:1575-84.

16 Egger HL, Angold A. Common emotional and behavioral disorders in preschool children: presentation, nosology, and epidemiology. $J$ Child Psychol Psychiatry 2006;47:313-37.

17 Al Ansari A, Hamadeh RR, Jahrami H, et al. Outcomes of children with attention deficit/hyperactivity disorder: global functioning and symptoms persistence. East Mediterr Health J 2017;23:589-93.

18 Fairchild G, van Goozen SHM, Calder AJ, et al. Research review: evaluating and reformulating the developmental taxonomic theory of antisocial behaviour. J Child Psychol Psychiatry 2013;54:924-40.
19 Dahl V, Ramakrishnan A, Spears AP, et al. Psychoeducation interventions for parents and teachers of children and adolescents with ADHD: a systematic review of the literature. J Dev Phys Disabil 2020;32:257-92.

20 Rueger SY, Katz RL, Risser HJ, et al. Relations between parental affect and parenting behaviors: a meta-analytic review. Parenting 2011;11:1-33.

21 Montoya A, Colom F, Ferrin M. Is psychoeducation for parents and teachers of children and adolescents with ADHD efficacious? A systematic literature review. Eur Psychiatry 2011;26:166-75.

22 Ferrin M, Moreno-Granados JM, Salcedo-Marin MD, et al. Evaluation of a psychoeducation programme for parents of children and adolescents with ADHD: immediate and long-term effects using a blind randomized controlled trial. Eur Child Adolesc Psychiatry 2014;23:637-47.

23 Lopez MA, Toprac MG, Crismon ML, et al. A psychoeducational program for children with ADHD or depression and their families: results from the CMAP feasibility study. Community Ment Health $J$ 2005;41:51-66.

24 Miranda A, Presentación MJ, Soriano M. Effectiveness of a schoolbased multicomponent program for the treatment of children with ADHD. J Learn Disabil 2002;35:547-63.

25 Monastra VJ. Overcoming the barriers to effective treatment for attention-deficit/hyperactivity disorder: a neuro-educational approach. Int J Psychophysiol 2005;58:71-80.

26 Svanborg P, Thernlund G, Gustafsson PA, et al. Efficacy and safety of atomoxetine as add-on to psychoeducation in the treatment of attention deficit/hyperactivity disorder: a randomized, double-blind, placebo-controlled study in stimulant-naïve Swedish children and adolescents. Eur Child Adolesc Psychiatry 2009;18:240-9.

27 Corkum P, Rimer P, Schachar R. Parental knowledge of attentiondeficit hyperactivity disorder and opinions of treatment options: impact on enrollment and adherence to a 12-month treatment trial. Can J Psychiatry 1999;44:1043-8.

28 Hack S, Chow B. Pediatric psychotropic medication compliance: a literature review and research-based suggestions for improving treatment compliance. J Child Adolesc Psychopharmacol 2001;11:59-67.

29 Mirza M, Krischer A, Stolley M, et al. Review of parental activation interventions for parents of children with special health care needs. Child Care Health Dev 2018;44:401-26.

30 Roughan LA, Parker JR, Mercer L. Improving interventions for parents of children and young people with autism spectrum disorder (ASD) in CAMHS. BMJ Open Qual 2019;8:e000261.

31 Kieckhefer GM, Trahms CM, Churchill SS, et al. A randomized clinical trial of the building on family strengths program: an education program for parents of children with chronic health conditions. Matern Child Health J 2014;18:563-74.

32 van Gestel-Timmermans H, Brouwers EPM, van Assen MALM, et al. Effects of a peer-run course on recovery from serious mental illness: a randomized controlled trial. Psychiatr Serv 2012;63:54-60.

33 Turner A, Anderson JK, Wallace LM, et al. An evaluation of a selfmanagement program for patients with long-term conditions. Patient Educ Couns 2015;98:213-9.

34 Sharma S, Wallace LM, Kosmala-Anderson J, et al. A process evaluation using a self determination theory measure of the codelivery of self management training by clinicians and by lay tutors. Patient Educ Couns 2013;90:38-45.

35 Elg M, Engström J, Witell L, et al. Co-creation and learning in healthcare service development. J Service Management 2012;23:328-43.

36 Stenberg U, Haaland-Øverby M, Fredriksen K, et al. A scoping review of the literature on benefits and challenges of participating in patient education programs aimed at promoting self-management for people living with chronic illness. Patient Educ Couns 2016;99:1759-71.

37 Lara-Cabrera ML, Gjerden M, Gråwe RW, et al. Short-term effects of a peer co-led educational programme delivered before mental health treatment: a randomised controlled trial. Patient Educ Couns 2016;99:1257-61.

38 Lara-Cabrera ML, Salvesen Øyvind, Nesset MB, et al. The effect of a brief educational programme added to mental health treatment to improve patient activation: a randomized controlled trial in community mental health centres. Patient Educ Couns 2016;99: 760-8.

39 Chacko A, Hopkins K, Acri M, et al. Expanding attention-deficit/ hyperactivity disorder service provision in urban socioeconomically disadvantaged communities: a pilot study. Clin Pract Pediatr Psychol 2020;8:189-94.

40 Chacko A, Scavenius C. Bending the curve: a community-based behavioral parent training model to address ADHD-Related concerns in the voluntary sector in Denmark. J Abnorm Child Psychol 2018;46:505-17. 
41 Stenberg U, Haaland-Øverby M, Koricho AT, et al. How can we support children, adolescents and young adults in managing chronic health challenges? A scoping review on the effects of patient education interventions. Health Expect 2019;22:849-62.

42 Edwards AG, Brebner CM, McCormack PF, et al. From 'parent' to 'expert': how parents of children with autism spectrum disorder make decisions about which intervention approaches to access. $J$ Autism Dev Disord 2018;48:2122-38.

43 Chesterfield JA, Porzig-Drummond R, Stevenson RJ, et al. Evaluating a brief behavioral parenting program for parents of school-aged children with ADHD. Parenting 2020:1-25.

44 Daley D, Van Der Oord S, Ferrin M, et al. Practitioner review: current best practice in the use of parent training and other behavioural interventions in the treatment of children and adolescents with attention deficit hyperactivity disorder. J Child Psychol Psychiatry 2018;59:932-47.

45 Sonuga-Barke EJS, Barton J, Daley D, et al. A comparison of the clinical effectiveness and cost of specialised individually delivered parent training for preschool attention-deficit/hyperactivity disorder and a generic, group-based programme: a multicentre, randomised controlled trial of the new forest parenting programme versus Incredible years. Eur Child Adolesc Psychiatry 2018;27:797-809.

46 Bell ML, Whitehead AL, Julious SA. Guidance for using pilot studies to inform the design of intervention trials with continuous outcomes. Clin Epidemiol 2018;10:153-7.

47 Julious SA. Pilot studies in clinical research. Stat Methods Med Res 2016;25:995-6.

48 Whitehead AL, Julious SA, Cooper CL, et al. Estimating the sample size for a pilot randomised trial to minimise the overall trial sample size for the external pilot and main trial for a continuous outcome variable. Stat Methods Med Res 2016;25:1057-73.

49 Chan A-W, Tetzlaff JM, Gøtzsche PC, et al. Spirit 2013 explanation and elaboration: guidance for protocols of clinical trials. BMJ 2013;346:e7586.

50 Schulz KF, Grimes DA. Allocation concealment in randomised trials: defending against deciphering. Lancet 2002;359:614-8.

51 Chan A-W, Tetzlaff JM, Altman DG, et al. Spirit 2013 statement: defining standard protocol items for clinical trials. Ann Intern Med 2013;158:200-7

52 DeCamp LR, Leifheit K, Shah $\mathrm{H}$, et al. Cross-Cultural validation of the parent-patient activation measure in low income Spanish- and English-speaking parents. Patient Educ Couns 2016;99:2055-62.

53 Fowles JB, Terry P, Xi M, et al. Measuring self-management of patients' and employees' health: further validation of the patient activation measure (PAM) based on its relation to employee characteristics. Patient Educ Couns 2009;77:116-22.

54 Hibbard JH, Mahoney ER, Stock R, et al. Do increases in patient activation result in improved self-management behaviors? Health Serv Res 2007;42:1443-63.
55 Attkisson CC, Zwick R. The client satisfaction questionnaire. psychometric properties and correlations with service utilization and psychotherapy outcome. Eval Program Plann 1982;5:233-7.

56 Bech $\mathrm{P}$, Olsen LR, Kjoller M, et al. Measuring well-being rather than the absence of distress symptoms: a comparison of the SF-36 mental health subscale and the WHO-Five well-being scale. Int J Methods Psychiatr Res 2003;12:85-91.

57 Mezzich JE, Cohen NL, Ruiperez MA, et al. The multicultural quality of life index: presentation and validation. J Eval Clin Pract 2011;17:357-64.

58 De las Cuevas C, Rivero-Santana A, Perestelo-Perez L, et al. Adaptation and validation study of the beliefs about medicines questionnaire in psychiatric outpatients in a community mental health setting. Hum Psychopharmacol 2011;26:140-6.

59 Jónsdóttir H, Friis S, Horne R, et al. Beliefs about medications: measurement and relationship to adherence in patients with severe mental disorders. Acta Psychiatr Scand 2009;119:78-84.

60 Horne R, Chapman SCE, Parham R, et al. Understanding patients' adherence-related beliefs about medicines prescribed for longterm conditions: a meta-analytic review of the Necessity-Concerns framework. PLoS One 2013;8:e80633.

61 De Las Cuevas C, de Leon J. Development and validation of the patient's health belief questionnaire on psychiatric treatment. Patient Prefer Adherence 2019;13:527-36.

62 Morán-Sánchez I, Gómez-Vallés P, Bernal-López María Ángeles, et al. Shared decision-making in outpatients with mental disorders: Patients' preferences and associated factors. J Eval Clin Pract 2019;25:1200-9.

63 Degner LF, Sloan JA, Venkatesh P. The control preferences scale. Can J Nurs Res 1997;29:21-43.

64 Barr PJ, Thompson R, Walsh T, et al. The psychometric properties of collaborate: a fast and frugal patient-reported measure of the shared decision-making process. J Med Internet Res 2014;16:e2.

65 Swanson JM, Kraemer HC, Hinshaw SP, et al. Clinical relevance of the primary findings of the MTA: success rates based on severity of ADHD and odd symptoms at the end of treatment. J Am Acad Child Adolesc Psychiatry 2001;40:168-79.

66 Kornør H, Bøe T. Måleegenskaper ved den norske versjonen av swanson, nolan og pelham vurderingsskala for lærere og foreldre versjon IV (SNAP-IV). PsykTestBarn 2011;1:1.

67 Lundh A, Kowalski J, Sundberg CJ, et al. Children's global assessment scale (CGAS) in a naturalistic clinical setting: interrater reliability and comparison with expert ratings. Psychiatry Res 2010;177:206-10.

68 Shaffer D, Gould MS, Brasic J, et al. A children's global assessment scale (CGAS). Arch Gen Psychiatry 1983;40:1228-31.

69 Schorre BEH, Vandvik IH. Global assessment of psychosocial functioning in child and adolescent psychiatry. A review of three unidimensional scales (CGAS, GAF, GAPD). Eur Child Adolesc Psychiatry 2004;13:273-86. 